\title{
Erratum to: Clinical and Neuropsychological Outcome After Microsurgical and Endovascular Treatment of Ruptured and Unruptured Anterior Communicating Artery Aneurysms: A Single-Enter Experience
}

Andrea Pietrantonio, Sokol Trungu, and Antonino Raco

M. Visocchi et al. (eds.), Chapter 27 in: Trends in Reconstructive Neurosurgery, Acta Neurochirurgica Supplement 124, DOI 10.1007/978-3-319-39546-3_27

The order of the author's names was wrong in chapter 27. The correct names are:

1) Andrea Pietrantonio

2) Sokol Trungu

3) Antonino Raco

The updated original online version for this chapter can be found at DOI 10.1007/978-3-319-39546-3_27 